\title{
A case of Takayasu's aortoarteritis with severe dilated cardiomyopathy in pregnancy
}

\author{
Meena Satia, Trupthi Ganapathi*, Shruti Panchbudhe
}

\begin{abstract}
Department of Obstetrics and Gynaecology, Seth Gordhandas Sunderdas Medical College and King Edward VII Memorial Hospital, Mumbai, Maharashtra, India
\end{abstract}

Received: 20 June 2016

Accepted: 09 July 2016

\section{*Correspondence:}

Dr. Trupthi Ganapathi,

E-mail: trupthi148@gmail.com

Copyright: (c) the author(s), publisher and licensee Medip Academy. This is an open-access article distributed under the terms of the Creative Commons Attribution Non-Commercial License, which permits unrestricted non-commercial use, distribution, and reproduction in any medium, provided the original work is properly cited.

\begin{abstract}
Takayasu's aortoarteritis (TA) is a idiopathic, rare, chronic, giant-cell inflammatory disease of the arteries which primarily involves the aorta, its main branches and coronary and pulmonary arteries Women are affected in $80-90 \%$ of cases with a mean age of presentation in the second and third decade of life, reflected in a synonym for TA as "young female arteritis". Also known as pulse less disease that causes progressive damage including inflammation, scarring, narrowing and abnormal ballooning inside the wall of aorta and major arteries. A pregnancy in such cases is often associated with severe life-threatening complications and is hazardous to the life of the mother and therefore requires contraception counseling. Our case is a coexistence of Takayasu's aortoarteritis and dilated cardiomyopathy which is an uncommon complication of TA and hence required termination of pregnancy.
\end{abstract}

Keywords: TA, Giant-cell inflammatory disease, Pregnancy

\section{INTRODUCTION}

Takayasu's aortoarteritis is a condition that causes narrowing, occlusion and aneurysm of medium and large arteries. It affects aorta and its branches, resulting in nonspecific aortoarteritis. ${ }^{1}$ This disease is commonly seen in females between $2^{\text {nd }}$ and $3^{\text {rd }}$ decade. Its incidence varies between 1.2 and 2.3 cases per million per year, and it is more common in Asians than in other racial groups. ${ }^{2}$ Various causes have been suspected in TA, initially it was considered an infection and later identified as autoimmune condition. Genetic factors have also found to play an important role. ${ }^{3}$ Management of patients with TA during pregnancy is a challenging task owing to the multiple cardiovascular complications that may occur in the course of the disease. Whether the status of the disease improves, remains unchanged, or worsens during pregnancy also need to be evaluated. Optimal management for pregnant patients with this disease has not yet been established therefore guidelines for the treatment of pregnant women with TA are necessary. ${ }^{4}$
There are only few case reports in the literature about women who were diagnosed with TA during pregnancy. The evolution of the disease is not affected by pregnancy however pregnant women with Takayasu's aortoarteritis can develop complications due to hindrance of regional blood flow; hypertension or multi organ dysfunction Pregnancy in Takayasu's aortoarteritis is often associated with severe life-threatening complications. Management of Takayasu's is challenging task for obstetrician, rheumatologist and cardiologist owing to the multiple complications associated with the disease. Dilated cardiomyopathy is an uncommon complication of TA with a prevalence of about $6 \% .^{5}$ Histopathological study of the myocardium in these patients has revealed nonspecific inflammation of myocardium with lymphocyte/mononuclear cell infiltration and normal coronary vessels. ${ }^{6}$ 


\section{CASE REPORT}

23 year old multigravida with history of 2 abortions presented at 6weeks gestation to OPD with complaints of breathlessness and claudication pain in both upper and lower limbs since 6months, she had no history of chest pain, palpitations, syncope or oedema of the feet. No history of tuberculosis in the past. She had been to a Physician with similar complaints where she was transfused 1pint of packed cell for severe anemia and was advised a CT Angiogram for limb claudication.

On examination her general condition was fair. Her pulse was 100/minute felt in left brachial artery. Both right and left radial artery pulsations were not felt and blood pressure was $90 / 60 \mathrm{mmHg}$ in both upper limbs. Bilateral Dorsalis pedis pulsations were feeble with blood pressure of $140 / 80 \mathrm{mmHg}$ in the lower limbs. In view of the above findings a cardiology reference was taken. There were no ischemic changes or neurological deficit in the limbs. Bilateral carotid pulsations were prominent. Cardiovascular and respiratory system and per abdomen examination was normal and per vaginum examination revealed uterus of 6 weeks size, anteverted, bilateral fornices free.

Her investigations were as follows: complete hemogram was normal, her renal function tests and liver function tests were within normal limits. Her ESR was raised (61), her CRP level was 50.1, autoimmune markers were negative (ANA, Anti dsDNA). Her chest $\mathrm{x}$-ray with abdominal shield was normal. ECG revealed left ventricular hypertrophy. Her CT angiogram showed diffuse wall thickening of ascending aorta, arch of aorta, descending, thoracic and abdominal aorta suggestive of Takayasu's aortoarteritis.

2D Echo revealed Global ventricular hypokinesia with moderate mitral regurgitation, mild aortic regurgitation, trileaflet aortic valve with ejection fraction $25 \%$ with left ventricular systolic dysfunction suggestive of severe cardiomyopathy. Her USG abdomen revealed mild splenomegaly. Her renal Doppler was normal. Cardiology and rheumatology opinion was taken. Patient was started on Tablet Prednisolone 20mg twice a day for 3 months followed by $40 \mathrm{mg}$ on alternate days and subsequently the dose was tapered, Tablet Furosemide $25 \mathrm{mg}$ once a day, Tablet Cardivas $3.125 \mathrm{mg}$ twice a day, Tablet Ramipril $2.5 \mathrm{mg}$ once a day and anti-tubercular drugs (category -1) was started.

Patient and relatives were counselled regarding the high risk associated with the condition High risk consent was taken. ICU stand by and ventilator was reserved for the patient. After Cardiology and Rheumatology fitness patient underwent medical termination of pregnancy by suction and evacuation method in view of grave injury to the physical health of mother. The procedure was uneventful. Post procedure patient was stable Antibiotic prophylaxis was given for seven days.

\section{DISCUSSION}

Takayasu aortoarteritis of the arteries was first noted in 1908 by Japanese Ophthalmologist Takayasu. This disease entity has been known by a variety of terms like pulseless disease, reversed coarctation occlusive aortopathy, aortic arch syndrome, and young female arteritis

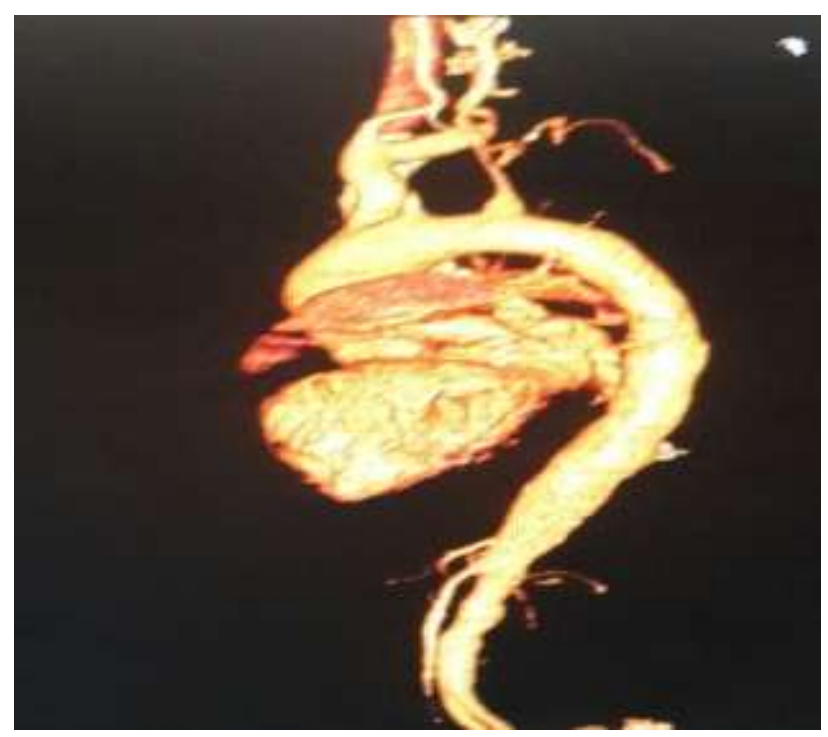

Figure 1: 3D image of aorta.

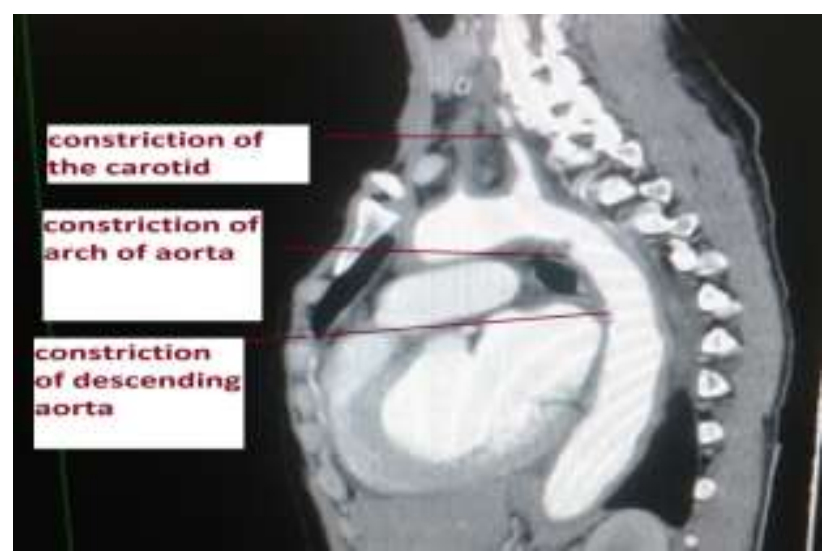

Figure 2: Computed tomography aortogram.

Although the exact mechanism behind this damage is unknown, research suggests that TA is stimulated by changes in the body's immune response. Most probably, TA is an autoimmune disorder, meaning that immune defences attack the body's own cells rather than outside invaders. ${ }^{7}$ Maternal morbidity may be high because the risk of aortic dissection, as well as of intra cerebral bleed from rupture of an aneurysm of the circle of Willis increases in pregnancy. Surgical correction before pregnancy reduces the risk of aortic complications during pregnancy, and also reduces the incidences of fetal complications, such as IUGR or congenital heart disease. The final outcome is also affected when valvular system abnormalities are noted and also by the associated 
complications with the disease. In uncomplicated cases usually the pregnancy and delivery are uneventful. Cesarean section is done as per obstetrician's decision, our case was complicated with severe dilated cardiomyopathy and hence pregnancy was terminated.

The criteria for the diagnosis of Takayasu's aortoarteritis consists of one obligatory criterion (age $\leq 40$ years), two major criteria (left and right mid subclavian artery lesions) and nine minor criteria (high erythrocyte sedimentation rate, common carotid artery tenderness, hypertension, aortic regurgitation or annuloaortic ectasia and lesions of the pulmonary artery, left mid common carotid artery, distal brachiocephalic trunk, thoracic aorta and abdominal aorta).

In addition to the obligatory criterion, the presence of two major criteria or of one major plus two or more minor criteria, or of four or more minor criteria suggests an increased probability of the presence of Takayasu's disease. ${ }^{8}$ The disease causes various clinical conditions such as painful arm due to claudication, decreased artery pulses, carotodynia, visual loss, stroke, aortic regurgitation, hypertension and congestive heart failure. Clinical presentation may be insidious, early symptoms of aortoarteritis usually are not specific and $96 \%$ of patients are not diagnosed until they suffer later symptoms related to decreased arterial blood flow. The most serious effect of aortoarteritis is a thickening and stenosis of affected arteries which may interfere with the normal blood flow to organs supplied by the stenosed vessels

Our patient had one obligatory criterion (age 23ys), and 5 minor criteria- high ESR, hypertension in lower limbs, aortic regurgitation, involvement of thoracic and abdominal aorta. Presence of dilated cardiomyopathy in a case of Takayasu's aortoarteritis is a common though not rare but rarely diagnosed.

A causal relationship between Takayasu's aortoarteritis and tuberculosis (TB) had been suggested as both the diseases show similar pathological changes, chronic inflammatory lesions and, occasionally, granulomas on the arterial walls. Both diseases have been associated with human leukocyte antigen (HLA) alleles, cryoglobulins and cold agglutinins during the acute phase of the illness but genetic relationship has not been recognised yet. Previous studies have shown that crossreaction between Mycobacterium and human heat shock protein (HSP) might have a role.

This hypothesis has been supported by the increase in expression of $65 \mathrm{KDa}$ HSPs of the involved vessels as well as the activation of $\mathrm{T}$ cells that may cross-react with the host HSP. It has been speculated that Mycobacterium tuberculosis can be the stimulating factor through its production of super antigens that induce vascular damage by the autoimmune $\mathrm{T}$ cells for which our patient was started on anti-tubercular drugs (category 1$){ }^{2}$ There are case reports where patient has responded well to prednisolone and anti TB drugs. ${ }^{9}$ The 15 -year survival was $66.3 \%$ and $96.4 \%$ with and without major complications. ${ }^{3}$

Treatment of TA is mainly corticosteroids and immunosuppressant's like Cyclophosphamide and Methotrexate have also been tried. If TA has caused stenosis of an artery, the narrowed segment may need to be dilated. Depending on the specific artery affected, this can be done either by open surgery or percutaneous trans luminal balloon angioplasty or placement of a stent -an implanted device that keeps the narrowed vessel open.. Surgery has low morbidity and mortality except in aortic aneurysm surgery.

\section{CONCLUSION}

Fertility potential is not affected and pregnancy does not appear to exacerbate the disease, Takayasu's aortoarteritis is an autoimmune disorder that requires proper guidelines for management during pregnancy and all such patients should have a medical screening prior to conception and anatomical pattern of the disease should be carefully evaluated. Medical management of pregnant patients with Takayasu aortoarteritis is similar to the non-pregnant patient. Multidisciplinary management is mandatory for good clinical outcome during pregnancy and their blood pressure should be strictly controlled for a positive maternal and perinatal outcome.

\section{Funding: No funding sources \\ Conflict of interest: None declared \\ Ethical approval: Not required}

\section{REFERENCES}

1. Hanna RM, Wan-Ting Y, Kim SJ, Lopez EA. Three Presentations of Takayasu's Arteritis in Hispanic Patients Case Reports, Medicine. 2012, Article ID 839795, 7 pages.

2. Al-Aghbari K, Al-Motarreb A. Takayasu's Arteritis Associated with Tuberculosis in a Young Yemeni Woman, Heart Views. 2010;11(3):117-20.

3. Ogino H. Heart Disease in Asia Overview of Late Outcome of Medical and Surgical Treatment for Takayasu Arteritis. 2008;118:2738-47.

4. Hauenstein E, Frank H, Bauer JS, Schneider KT, Fischer T. Takayasu's arteritis in pregnancy: review of literature and discussion. $\mathrm{J}$ Perinat Med. 2010;38(1):55-62

5. Patra S, Sastry UM, Mahimaiha J, Subramanian AP, Shankarappa RK, Nanjappa MC. Dilated cardiomyopathy being the presenting manifestation of Takayasu arteritis and treated with renal angioplasty. World J Pediatr Congenit Heart Surg. 2014;5(4):620-2.

6. Ghosh S, Sinha DP, Ghosh S, Mitra D. Indian Heart $\mathrm{J}$ Dilated cardiomyopathy in non-specific aortoarteritis. 1999;51(5):527-31. 
7. Choudhari KR. Aortoarteritis in pregnancy. Bombay Hospital Journal. 2002.

8. Ishikawa K. Diagnostic approach and proposed criteria for the clinical diagnosis of Takayasu's arteriopathy; Journal of the American College of Cardiology. 1988;12(4):964-72.

9. Baijal R, Chogle A. A Case of Tuberculous Colitis with Associated Takayasu's Arteritis. Journal of the association of physicians of India. 2015;63.
Cite this article as: Satia M, Ganapathi T, Panchbudhe S. A case of Takayasu's aortoarteritis with severe dilated cardiomyopathy in pregnancy. Int J Reprod Contracept Obstet Gynecol 2016;5:2884-7. 